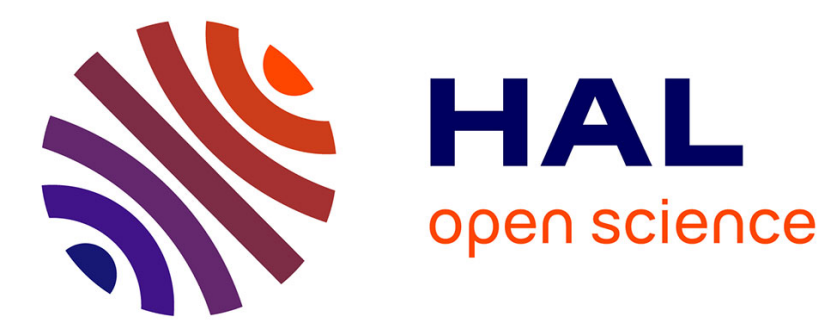

\title{
Forêts domaniales et politique forestière néerlandaise depuis 1945. Evolution et résultats
}

\author{
A.J. Grandjean
}

\section{To cite this version:}

A.J. Grandjean. Forêts domaniales et politique forestière néerlandaise depuis 1945. Evolution et résultats. Revue forestière française, 1987, 39 (3), pp.219-230. 10.4267/2042/25786 . hal-03424758

\section{HAL Id: hal-03424758 \\ https://hal.science/hal-03424758}

Submitted on 10 Nov 2021

HAL is a multi-disciplinary open access archive for the deposit and dissemination of scientific research documents, whether they are published or not. The documents may come from teaching and research institutions in France or abroad, or from public or private research centers.
L'archive ouverte pluridisciplinaire HAL, est destinée au dépôt et à la diffusion de documents scientifiques de niveau recherche, publiés ou non, émanant des établissements d'enseignement et de recherche français ou étrangers, des laboratoires publics ou privés. 


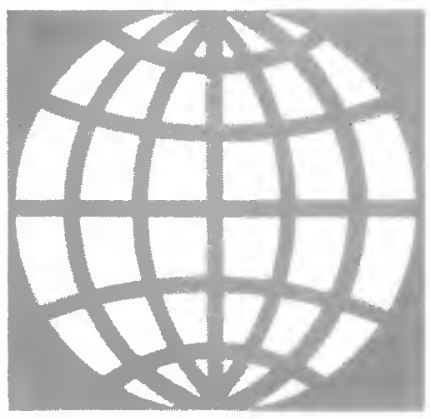

\section{chronique internationale}

\section{FORÊTS DOMANIALES ET POLITIQUE FORESTIÈRE NÉERLANDAISE DEPUIS 1945 ÉVOLUTION ET RÉSULTATS}

\section{A.J. GRANDJEAN}

Au terme d'une carrière de quarante ans au ministère de l'Agriculture et de la Pêche, dont dépend l'Administration forestière néerlandaise (AFN), c'est à la fois tentant et difficile de dresser un tableau de l'adaptation de la gestion des forêts de l'Ėtat aux développements politiques et sociaux du pays.

Le Royaume des Pays-Bas, ordinairement appelé Hollande - synonyme du mot Holtland : pays boisé - occupe un territoire de 3,4 millions d'hectares avec une population de 14,5 millions d'habitants et une superficie boisée de 330000 hectares. Bien que cette superficie forestière ne représente que $9 \%$ du territoire ou $235 \mathrm{~m}^{2}$ par habitant, le pays donne l'impression d'être très boisè. C'est que les forêts sont fort morcelées, et presque partout il y a des plantations le long des routes et des canaux, beaucoup de rideaux d'arbres, représentant au total une longueur de 45000 kilomètres, équivalente à 35000 hectares de forêts.

Les ètudes paléobotaniques montrent qu'anciennement le pays plat - delta des grands fleuves du Rhin et de la Meuse - était densément boisé d'essences feuillues sur les sols humides ou secs, sur les marais d'eau saumâtre ou douce. Depuis le Moyen Age, ces forêts ont été mises en valeur comme terres agricoles, ou dégradées en landes à bruyères, sables et tourbières. Au début du XIXème siècle, la superficie forestière fut au plus bas : 50000 hectares de futaie et 120000 hectares de taillis. La crise agricole, à la fin du XIXème siècle, a motivé un reboisement de terres agricoles, suivi d'un boisement des landes à bruyères pendant le grand chômage des années 1929-39. Par la suite, les forêts résineuses ont été cantonnées sur les sols sableux secs

* Monsieur Grandjean, ingẻnıur forestier diplómé de l'université agronomique de Wageningen, directeur adjoint de l'Administration forestière néerlandaise de 1963 a 1972 , a terminè sa carrière comme directeur de la province de la Gueldre pour le ministére de l'Agriculture et de la Pêche. II était alors chargè de tout l'éventail des actions existant en zones non urbaines, y compris celles relatives à la qualitè de l'environnement. 


\section{A.J. GRANDJEAN}

de mauvaise qualité comme les dunes, les sables mouvants et les podzols. Exceptionnellement, il y a des forêts sur sols riches et argileux, sur sols à loess et sur dépôts morainiques humides : il s'agit surtout de peuplements d'Epicéa et Mèlèze du Japon et aussi de Chêne, de Hêtre et de Peuplier.

Après cet aperçu historique, il est facile de comprendre qu'il n'y a pas une tradition forestière enracinée dans la population néerlandaise. Pendant la guerre, il y avait 250000 hectares de forêts aux Pays-Bas. Durant les quarante dernières années, cette superficie a été étendue à 330000 hectares. Le tableau I détaille cette importante progression.

Tableau I Statistique forestière sur une période de $\mathbf{4 0}$ ans (en chiffres ronds)

\begin{tabular}{|c|c|c|c|c|}
\hline \multirow{2}{*}{$\begin{array}{l}\text { Type de forêt } \\
\text { Futaie resineuse } \ldots \ldots \ldots \ldots \ldots \ldots \\
\text { Futaie feuillue } \ldots \ldots \ldots \ldots \ldots \ldots \ldots \\
\text { Taillis et oseraies } \ldots \ldots \ldots \ldots \ldots \ldots\end{array}$} & \multicolumn{2}{|c|}{ Inventaire 1938-1942 } & \multicolumn{2}{|c|}{ Inventaire 1980-1983 } \\
\hline & $\begin{array}{c}174000 \text { ha } \\
28000 \\
48000\end{array}$ & $\begin{array}{l}70 \% \\
11 \\
19\end{array}$ & $\begin{array}{c}198000 \text { ha } \\
112000 \\
22000\end{array}$ & $\begin{array}{l}60 \% \\
34 \\
6\end{array}$ \\
\hline Forêt totale $\ldots \ldots \ldots \ldots \ldots \ldots \ldots$ & 250000 & 100 & 332000 & 100 \\
\hline $\begin{array}{l}\text { Propriête } \\
\text { Forêt domaniale } \ldots \ldots \ldots \ldots \ldots \ldots \\
\text { Autre forêt publique } \ldots \ldots \ldots \ldots \\
\text { Forêt privée } \ldots \ldots \ldots \ldots \ldots \ldots \ldots \\
\text { Forêt de fondations, sociètès, etc. }\end{array}$ & $\begin{array}{l}37000 \text { ha } \\
38000 \\
162000 \\
13000\end{array}$ & $\begin{array}{l}15 \% \\
15 \\
65 \\
5\end{array}$ & $\begin{array}{l}104000 \text { ha } \\
55000 \\
136000 \\
37000\end{array}$ & $\begin{array}{l}31 \% \\
17 \\
41 \\
11\end{array}$ \\
\hline Forêt totale .... & 250000 & 100 & 332000 & 100 \\
\hline
\end{tabular}

En ce qui concerne la distribution des essences, le tableau II prècise les changements considérables intervenus en trente ans. La "futaie totale " de ce tableau soustrait des donnèes "forêt totale " du tableau I les forêts non productives et non susceptibles d'un aménagement normal. On notera que la superficie des essences feuillues a augmenté considérablement, et que la superficie des essences rèsineuses s'est simplement maintenue.

Tableau II Répartition par essences sur une période de 30 ans (en chiffres ronds)

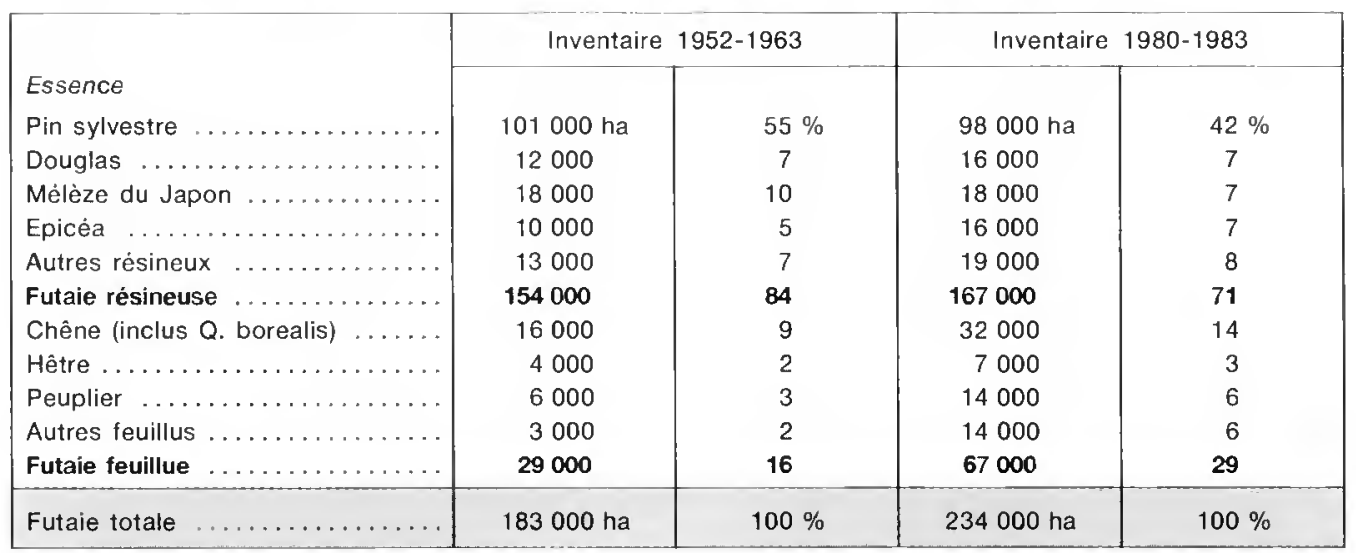

L'évolution de la gestion des forêts domaniales durant les quarante dernières annèes permet de distinguer quatre grandes périodes:

- 1945-1960 : restauration des forêts et production de bois prioritaires; 
- 1960-1970: promotion des loisirs en forêt ;

- 1970-1980: promotion de la protection de la nature;

- depuis 1980 : synthèse des buts précédents.

Ces périodes seront détaillées ci-après.

\section{RESTAURATION DES FORÊTS ET PRODUCTION DE BOIS}

A la fin de la Seconde Guerre mondiale, il y avait plusieurs dizaines de milliers d'hectares de forêts coupès ou très endommagés aux Pays-Bas, notamment une grande partie des forêts domaniales. La politique du gouvernement visait à la restauration de la productivité de l'agriculture pour l'alimentation de la population affamée. Dans ce cadre, la restauration des forêts était une priorité. La période 1945-1960 a été caractérisée par la poursuite de cet objectif, avant tout pour la production de bois. Par exemple, la conversion des taillis en futaie fut stimulee. Les plans d'aménagement visaient avant tout à l'augmentation de la production ligneuse et l'intérêt des essences exotiques à haute production fut mis en avant.

L'Institut national de Recherches forestières, appelé de Dorschkamp à Wageningen, se conformait à cette politique :

- développement des tables de production;

- recherche sur les essences exotiques, comme le Douglas et le Mélèze du Japon;

- amélioration de certaines essences, comme les Peupliers euraméricains ;

- lutte contre les dégâts des insectes, champignons, etc...

- emploi des engrais pour la stimulation de la croissance des essences, y compris recherches sur les relations entre la station forestière et les essences.

En ce qui concerne la sylviculture, avant la Guerre, beaucoup de forêts de la première génération de Pin sylvestre - l'essence la plus importante du pays - ont été créées par semis direct. Après 1945, cette méthode n'a plus été que très rarement employée. Elle fut remplacée par la plantation en lignes, une méthode plus sûre dans les conditions néerlandaises. La plantation à la main est applicable dans une forêt encombrée de rémanents. Cette méthode a été remplacée par la plantation mécanique, après qu'il fût possible de pulvériser les rémanents par broyage mécanique. Exceptionnellement, on a continué à boiser selon la méthode de l'ensemencement naturel, après une bonne année à semences. Hélas, ces bonnes années sont rares pour certaines essences comme le Douglas. Au fil des années, le reboisement mélangé par lignes a été remplacé par un mélange des essences en petits groupes, qui donne de meilleurs rèsultats.

Concernant l'espacement à la plantation, l'évolution a conduit à diminuer les nombres de plants à l'hectare, rendue possible par une meilleure qualité des graines. On a sélectionné des peuplements porte-graines de Pin sylvestre, Douglas et Mélèze du Japon ; I'AFN a ainsi disposè de graines de bonnes provenances et de haute qualité par les vergers à graines. L'Institut national de Recherches forestières a fait un travail excellent dans le domaine des provenances.

En ce qui concerne les nombres de plants à l'hectare, l'èvolution a par exemple été la suivante:

\begin{tabular}{|c|c|c|}
\hline & avant & après \\
\hline Pin sylvestre & 10000 plants & $2500-4500$ plants \\
\hline Douglas/Mèlèze $\ldots . . . \ldots \ldots \ldots . . . . .$. & 4000 plants & $2500-3000$ plants \\
\hline
\end{tabular}


Les Peupliers euraméricains ont toujours été plantés à 625 plants par hectare si une éclaircie est prévue, et à 200 plants sans éclaircie. Fréquemment, on a fait une plantation intercalaire d'Alnus incana pour protéger et enrichir le sol et pour améliorer le microclimat.

Avant et après la Guerre, sur sols pauvres, on a fait des mélanges "verticaux " dans certaines forêts de pins sylvestres âgés de plus de 30 ans par une plantation en sous-étage de Prunus serotina et/ou Quercus borealis, afin que l'humification soit améliorée. Après 20 ans, on a constaté de grands problèmes de concurrence de racines avec le Pin sylvestre. Plusieurs peuplements dépérissaient. En urgence, on s'est attaqué à ces sous-étages, en en frottant et contaminant les souches avec des produits chimiques (glycophosphate ou ammoniumsulphanate) sur plusieurs milliers d'hectares.

La coupe à blanc était la méthode usuelle de régénération des forêts de Pin sylvestre. Concernant les Pins ou autres essences de pleine lumière, cette méthode a été maintenue. Après la Guerre, elle a été remplacée par la coupe d'abri et la coupe en coulisses en cas de reboisement par du Douglas. Au début, I'AFN a privilégié la coupe d'abri avec des risques de dégâts graves pour la jeune régénération lors de l'exécution des coupes. A quoi s'ajoutent les dégâts de Hylobius et de Phomopsis sur Douglas après dommages mécaniques aux jeunes plants. Peu à peu, l'AFN a opté pour la coupe en coulisses qui a l'avantage de protéger la jeune culture en bandes, jusqu'à ce que la futaie restante soit coupée.

L'Institut national de Recherches forestières a constaté que les jeunes plants de Douglas sont très sensibles aux gelées tardives combinées avec l'ensoleillement fort pendant les mois de février et de mars. Le phénomène de dessèchement causé par l'èvaporation non compensée est très nuisible aux jeunes reboisements de Douglas. On peut éviter ces dégâts en plantant le Douglas dans des bandes d'une largeur d'une fois et demie la hauteur de la futaie de Pin sylvestre, de préférence orientées est-ouest. Les "coulisses " restent pendant 10-12 ans, après quoi leur futaie sera coupée entièrement, et les nouvelles bandes nues seront reboisées avec le Douglas. Cette méthode de la coupe en coulisses pour transformer la forêt de Pin en « douglasaie " est une méthode typique des Pays-Bas, développée depuis 1950.

La méthode de coupe par groupes a été appliquée surtout dans les vieilles forêts. II s'agit de forêts âgẻes mélangées de Chêne, Hêtre et essences résineuses, qui se reboisent régulièrement suivant des periodes de longue durée.

La méthode de coupe jardinée par pied d'arbre ne s'applique pas au pays. II y a eu des expériences restreintes dans un cantonnement, mais elles ne donnèrent pas de résultats satisfaisants.

Les données globales relatives aux quinze années précédentes sont les suivantes selon l'inventaire forestier de 1980-83:

- régénération par coupes à blanc: 22000 ha;

- régénération par coupes d'abri: 2000 ha ;

- régénération par coupes en coulisses : 700 ha ;

- régénération par coupes par bouquets : 200 ha.

Pendant la période 1945-1960, la production nationale de bois se vendait facilement parce que la demande surpassait l'offre. Il y avait une grande demande pour les mines de charbon, la reconstruction des fermes endommagées et pour l'usage agricole. Jusqu'à 1960, les bons prix des bois et les frais de production bas, ainsi que les salaires modiques des ouvriers forestiers, motivèrent une exploitation financière lucrative. Pour la forêt privée, les résultats financiers furent particulièrement avantageux grâce au système fiscal néerlandais, qui donne une tranchise de l'impôt sur les revenus des bois. Après 1960, la situation financière s'aggrava vite pour plusieurs raisons: 


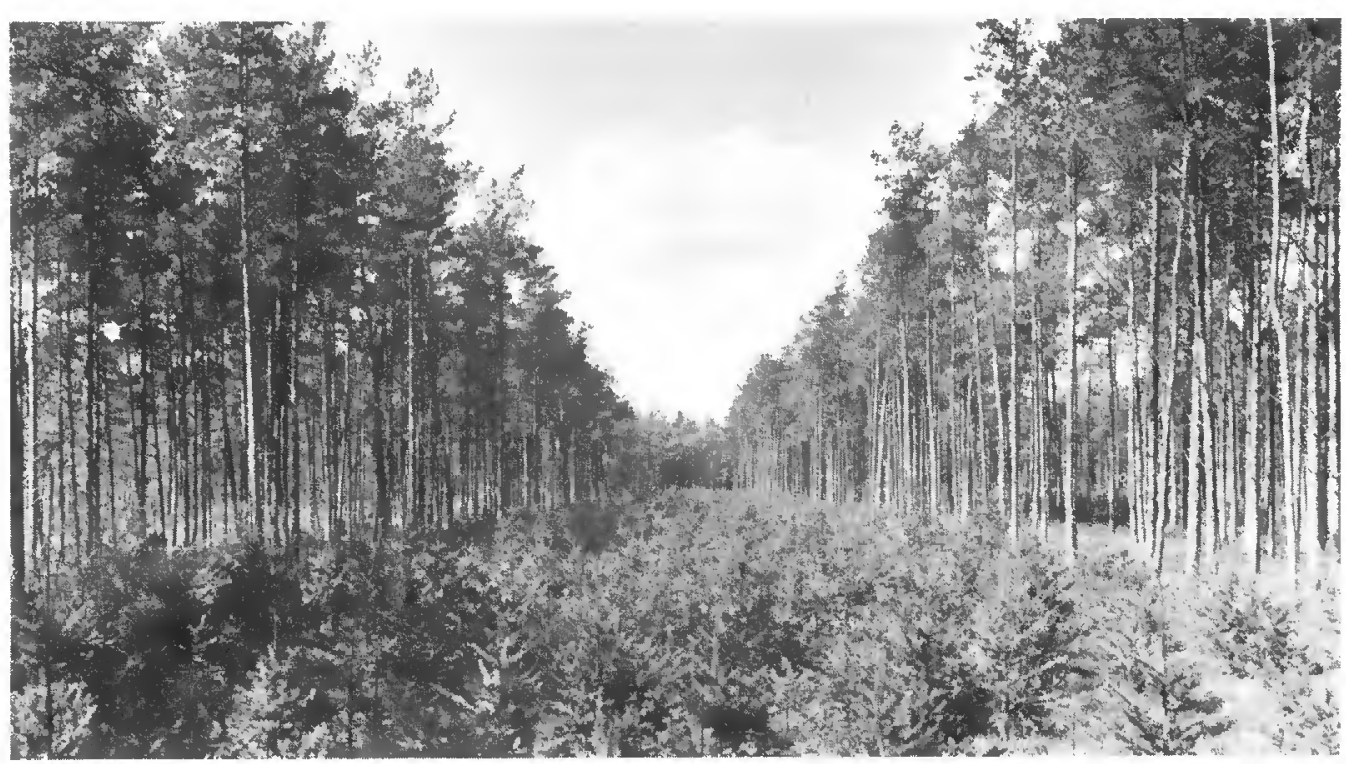

Coupes en coulisses et reboisement par du Douglas. Pins sylvestres de 65 ans et Douglas de 8 ans.

- les prix des bois de mines chutèrent considerablement du fait de la fermeture des mines de charbon dans le Limbourg pendant la période 1965-1973;

- les ventes au monde agricole diminuèrent;

- la concurrence des bois d'importation à bon marché se tit vive ;

- les salaires ouvriers et les frais généraux augmentèrent rapidement.

Le tableau III donne une idée du développement divergent des prix moyens des bois et des salaires des ouvriers forestiers.

Tableau III

Indices des prix des bois et des salaires d'ouvriers forestiers (en chiffres ronds)

\begin{tabular}{|c|c|c|c|}
\hline & Bois sur pied & Bois abattu & $\begin{array}{c}\text { Salaire + charges sociales } \\
\text { par heure productive }\end{array}$ \\
\cline { 2 - 4 } 1960 & 100 & 100 & 100 \\
1965 & 110 & 100 & 250 \\
1970 & 110 & 110 & 350 \\
1975 & 160 & 120 & 750 \\
1980 & 180 & 160 & 1000 \\
\hline
\end{tabular}

En 1950, environ 600000 mètres cubes furent produits; en 1980, la production de bois atteignit un million de mètres cubes. La production complémentaire des plantations hors forêt se montait à 100000 mètres cubes. Cette production nationale représentait alors $8 \%$ de la consommation nationale.

Malgré une recherche intensive de nouveaux débouchés à l'intérieur ou à l'exportation, malgré la rationalisation et la mécanisation des travaux forestiers, malgré encore les efforts de formation de groupements de vente et de travail entre les propriétaires privés, la situation des entreprises forestières resta défavorable. Le solde négatif des forêts domaniales monta à plus de 350 florins (= 1000 francs) par hectare en 1980 . Quant aux entreprises forestières privées, pour la moitié 


\section{A.J. GRANDJEAN}

seulement l'exploitation resta positive. Devant l'alarme des propriétaires privés, le gouvernement a institué une subvention au reboisement en 1969. Ce développement a aussi stimulé l'idée des forêts privées à buts multiples, comme proposé au 5ème Congrès forestier mondial de Seattle. A partir de 1966 (et mieux en 1976), l'État donna aux propriétaires une subvention pour le maintien et l'ouverture de leurs forêts aux promeneurs qui cherchent des loisirs dans les forêts privées. Ainsi la politique de restauration forestière par augmentation de la production de bois s'est transformée graduellement. L'accent fut mis sur les autres fonctions de la forêt comme les loisirs de plein air, la protection de la nature et l'importance des forêts pour la conservation de la beauté du paysage national.

\section{PROMOTION DES LOISIRS EN FORÊT}

Après 1960, avec l'augmentation du temps libre (semaine de travail de 40 heures, samedi libre, temps de vacances accru) ef l'augmentation du niveau de vie et de la mobilité de la population, les forêts nationales devenaient plus importantes pour les loisirs. L'intérêt porté par les partis politiques et les organismes de tourisme aux possibilités de récréation au pays augmentait.

II y eut quelques voyages d'études aux États-Unis pour étudier la politique d'installation et de gestion des parcs nationaux. La direction de l'AFN fonda une section centrale pour stimuler les possibilités de loisirs en forêts domaniales.

L'ouverture des forêts domaniales est fondée sur trois principes:

- l'entrée de tous les chemins et sentiers forestiers est libre:

- l'accès à l'intérieur des peuplements est interdit ;

- la création de zones pour les visiteurs, différentes des zones réservées à la production de bois et à la conservation de la nature est décidée; ces deux dernières donnent la possibilité de lieu de repos pour le grand gibier.

Quant au tracé des routes "touristiques" à travers les torêts domaniales, la possibilité de combinaison des chemins de loisirs et des chemins de débardage de bois est recherchée. Toute l'infrastructure mise en place par les cantonnements est basée sur ces trois principes.

Au cantonnement de Nunspeet par exemple, situé au nord de la région forestière de Veluwe, ce principe a été appliqué comme indiqué sur la carte.

Un plan d'aménagement à long terme prévoyait la création :

- d'une base d'amenagement récréatif et touristique en forêts par la création de zones de loisirs :

- de places de parking et de places de camping; il y a deux types de camping: de grandes places pour concentrer des visiteurs ordinaires, et de petites places pour les quelques visiteurs ayant un grand intérêt pour la nature;

- de places de concentration du public, comme des étangs et des aires de jeux;

- de routes touristiques pour la circulation motorisée en forêt et dans les régions à sites renommés ;

- de sentiers balisés de poteaux indicateurs, accompagnés de brochures avec carte et texte (livret) ;

- de centres d'information pour les visiteurs, y compris des guides qui dirigent les visiteurs au long de promenades instructives;

- de pistes cyclables touristiques et de pistes cavalières. 



\section{A.J. GRANDJEAN}

\section{PROMOTION DE LA PROTECTION DE LA NATURE}

Le mouvement pour la protection de la nature aux Pays-Bas avait commencé déjà avant la Guerre par la protection de certaines espèces rares de plantes et d'animaux. Après 1960, ce mouvement a évolué vers la protection du milieu et de la biocénose. L'année 1970 - année européenne pour la conservation de la nature du Conseil de l'Europe - fut stimulante. Adeptes de l'expression de François-René de Chateaubriand "Les forêts précèdent les peuples, les déserts les suivent", beaucoup d'associations et de particuliers soutiennent la protection de la nature et ont donné plus d'attention à la conservation des forêts et des réserves naturelles. Cette tendance a été soutenue par la prospérité nationale pendant la période 1965-1980. En politique, on a donné davantage d'intérêt aux choses immatérielles; un exemple clair, dans le cadre du "plan du delta", la décision politique extraordinairement importante en 1970 de construire un barrage perméable pour la sécurité de la Zélande et le maintien de la qualité du milieu salin de "l'Escaut de l'Est", avec flux et reflux maintenus.

En ce qui concerne la gestion des forêts néerlandaises, les différents mouvements de protection de la nature négligent la production de bois. Le maintien équilibré de la gestion des forêts domaniales par l'AFN était exposé à une certaine pression politique par ces mouvements actifs, suivis par quelques partis politiques. Un de ces mouvements appelé "le groupement de la sylviculture critique " développe une propagande pour l'abandon de l'aménagement de toutes les forêts - domaniales et privées - , pour le développement des valeurs naturelles des forêts et pour la diminution des frais de gestion; on obtenait ainsi, disait-on, une plus-value naturelle et économique, au lieu d'un déficit financier.

A l'ancien ministère de la Culture et des Affaires culturelles, ces groupements ou mouvements trouvèrent quelques soutiens, mais au ministère de l'Agriculture, y compris l'AFN, on eut une attitude réservée. Néanmoins l'idée a eu un résultat positif : cela stimula l'étude de nouvelles méthodes d'aménagement pour diminuer les frais de gestion usuels trop élevés. Des discussions violentes se produisirent parce que l'idée était contraire aux mœurs professionnelles de presque tous les ingénieurs torestiers praticiens. Les propriétaires privés aussi, ainsi que leur association nationale, ont pris leur distance vis-à-vis de cette conception: dans toutes les entreprises forestières, on reste convaincu de la nécessité d'une récolte annuelle de bois pour avoir un bilan financier convenable.

Depuis quelques années, il y a des discussions intenses entre les ingénieurs forestiers et les biologistes/écologistes sur la question de la réintroduction de grands animaux en forêt comme le loup, le bison et l'ours dans la région forestière centrale du pays (le Veluwe). Quelques expériences ont suivi, menées par l'AFN, la Société privée nationale de la protection de la nature, et les fondations provinciales. Des bovins d'Écosse, des chevaux polonais et des poneys d'Islande ont été introduits dans de petits parcs grillagés forestiers et aussi dans quelques landes à bruyères. Les résultats de ces expériences sont suivis avec grand intérêt par les forestiers praticiens. L'Institut national de l'Aménagement de la Nature est chargé du suivi scientifique. Les premiers résultats montrent que la végétation sous les arbres s'enrichit avec une plus grande diversité de plantes sur les places pâturées et les places à excréments.

Un autre aspect des chocs d'opinion entre les forestiers et les écologistes concerne la chasse en forêt. Les écologistes voudraient prohiber toute chasse, pour des raisons éthiques et pour la promotion de la régulation biologique dans un système naturel. La Société nationale pour la Conservation de la Nature, ayant une propriété totale de presque 70000 hectares de forêts et de réserves naturelles, a refusé de louer la chasse dans ses domaines. Cette règle est maintenant la base de la gestion de ces forêts, à moins que la chasse soit reconnue localement nécessaire pour la conservation des forêts et réserves naturelles (ce qui est exceptionnel). D'autres fondations provinciales de protection de la nature persistent à lover la chasse dans leurs forêts pour réguler l'équilibre de la faune. 
Avant 1960, la chasse dans les forêts domaniales était sous la responsabilité de l'AFN. Exceptionnellement, la chasse n'était pas louée dans quelques terrains domaniaux de grande valeur naturelle et scientifique. Depuis lors, une nouvelle direction centrale, compétente en matière de faune et de chasse a été creée au ministère de l'Agriculture. Cette direction loue la chasse dans les forêts domaniales, après consultation de l'AFN.

II y a quelques années, la direction de l'AFN a décidé d'installer plusieurs réserves forestières (au total 75) pour promouvoir la protection de la nature. Ces réserves boisées sont situées dans certains cantonnements répartis dans tout le pays. Elles ont chacune une superficie limitée (5 à 40 hectares), et sont entourées par des zones-tampon afin que les influences extérieures n'aient pas de mauvaises conséquences sylvicoles. La gestion de ces réserves est strictement limitée à la surveillance, sans aucune action forestière technique. Les cinq premières réserves ont déjả eté créées par décret ministériel.

Le premier pont spécialisé pour le passage des cerfs au-dessus d'une nouvelle autoroute qui traverse la region boisée centrale du Veluwe (appele "cerviduc") est en voie de construction. Voilà une nouveauté intéressante due à l'encouragement par les organisations pour la protection de la nature et du cerf indigène, et aussi par l'Association royale des chasseurs. La définition et l'achèvement du "cerviduc " est le résultat de la bonne coopération entre l'AFN, la Direction pour la nature et la faune du ministère de l'Agriculture et le service des Ponts-et-Chaussees. Un deuxième cerviduc sera construit sur la même autoroute l'année prochaine. La consultation avec les propriétaires concernés par le déplacement en recul corrélatif de leur engrillagement est en cours.

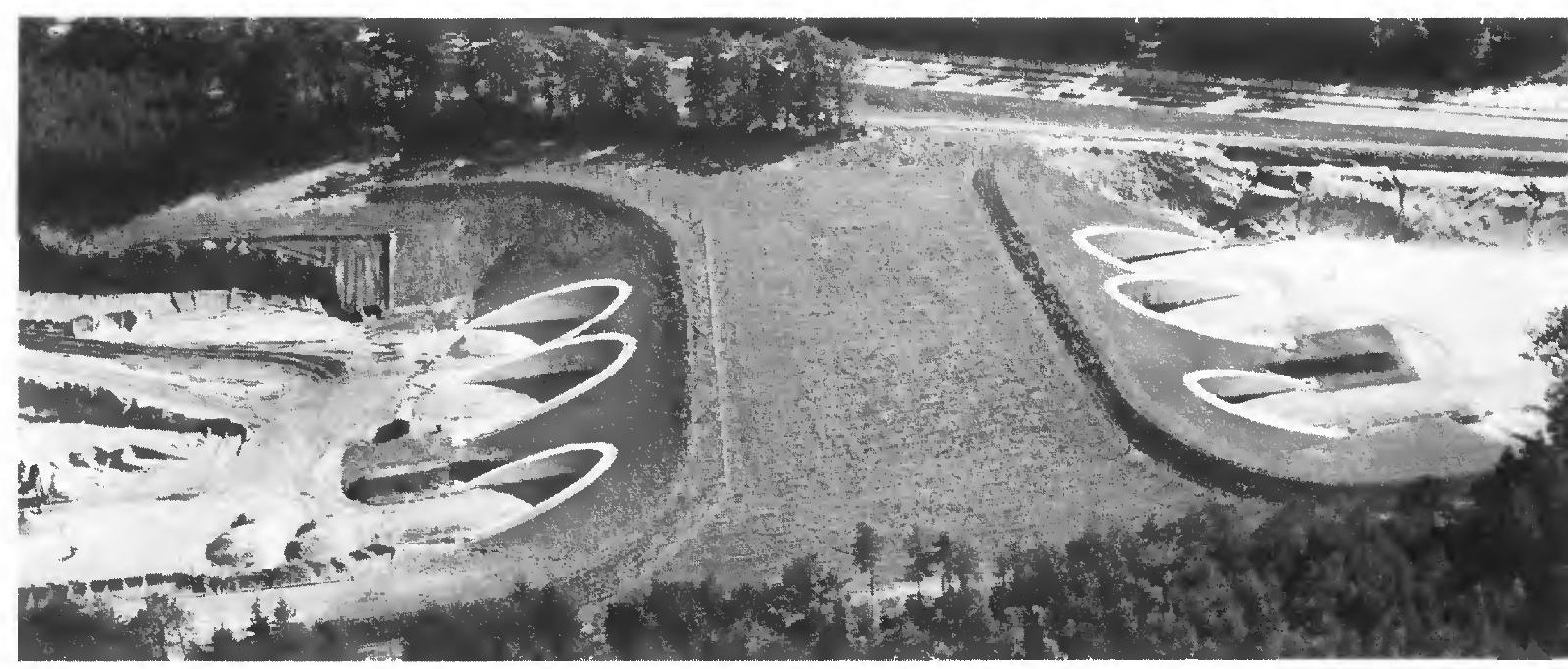

Cerviduc en construction. II sera planté ultérieurement. Les cerfs passeront au-dessus des trois tunnels de circulation. Nouvelle route nationale entre Arnhem et Apelddorn en construction (l'ancienne route, à l'arrière-plan, disparaîtra).

AEROPHOTO TEUGE - BRUMMEN

\section{LA SYNTHĖSE}

En 1976, le gouvernement a rendu public trois projets à long terme concernant:

- le génie rural, y compris le remembrement;

- le developpement des loisirs;

- la protection de la nature. 
Une procédure d'information du public, des organisations et des experts intéressés fut engagée, suivie par des consultations des Conseils provinciaux et autres corps publics; beaucoup de commentaires furent reçus. Après discussion au Parlement, le gouvernement prit ses décisions en 1985. Elles tèmoignent d'un grand intérêt pour la sylviculture nationale.

En 1984, un quatrième projet de plan à long terme a vu le jour. C'est un plan qui porte une attention spéciale à la politique forestière à venir. La même procédure aboutit à un plan définitif en 1986 qui contient la politique forestière du gouvernement pour la période 1986-2000, et même les années suivantes. Le but principal de ce plan est la promotion des conditions optimales pour la qualité des forêts dans le cadre de la communauté nationale.

L'ensemble de ces quatre plans traduit clairement la politique du gouvernement concernant l'ensemble futur des espaces verts, y compris l'agriculture, la sylviculture et le milieu rural. Cette politique jette aussi la base de l'aménagement provincial du territoire rural.

Le quatrieme plan concernant la sylviculture est le plus intéressant pour les lecteurs de la Revue forestière française. Les objectifs poursuivis se classent en cinq catégories :

- l'extension du territoire boisé : 30000 à 35000 hectares en plus environ ;

- l'augmentation de la production ligneuse: actuellement une production annuelte de un million de mètres cubes, en 2000 une production doublèe, et en 2030 une production de trois millions de mètres cubes;

- la consolidation de la protection de la nature avec $18 \%$ de la superficie nationale forestière réservée à la conservation de la nature; ce qui veut dire que ces forêts acquèreront une haute qualité écologique et ne seront pas destinées à produire du bois ;

- l'augmentation des possibilités de loisirs en plein air dans les forêts actuelles, et la plantation de 7000 hectares de forêts de loisirs dans de nouvelles zones, y compris 3000 hectares de parcs. Ces nouvelles forêts et parcs ont été programmés dans l'Ouest du pays où la population est concentrée dans le triangle Amsterdam-Rotterdam-Utrecht ;

- l'amélioration de la qualité du paysage, y compris la conservation d'une agriculture moderne qui doit pouvoir se développer.

Pour la réalisation de ces buts politiques futurs, le gouvernement se propose de constituer des plans de gestion de forêts régionaux basès sur la loi forestière (1961), qui devra être adaptée à cette intention. Ce plan régional sera compatible avec le plan d'aménagement rural, sous la responsabilité du Conseil provincial. Le plan régional contiendra la fixation des types futurs de forêt et des buts de gestion de la région forestière. II sera la base des plans de gestion concrets des diverses entreprises forestières. La cooperation des propriétaires privés sera obtenue par volontariat. L'AFN stimulera la réalisation des plans régionaux, grâce à sa connaissance spécifique des cantonnements forestiers du pays.

II est indispensable que les cinq buts du plan forestier du gouvernement soient appuyés par un budget annuel suffisant pour les mettre en œuvre dans une première période de 15 ans et après l'annèe 2000. Dans le triangle des trois métropoles, le boisement de terrains agricoles a commencé dans le cadre d'un projet spécial. Il y a quelques années, des remembrements agricoles accompagnés de plantations d'arbres ont aussi commencé. Bientôt une nouvelle superficie de 1250 hectares sera boisèe afin de réaliser un nouveau cantonnement avec plusieurs objectifs de loisirs, c'est-à-dire des forêts et parcs avec beaucoup de lisières appréciées des visiteurs. C'est une circonstance particulièrement heureuse que ce boisement, de plus en plus, s'accorde avec la politique de la CEE de réduire le territoire agricole pour diminuer les grands surplus de la production agricole de la CEE. La reine Béatrix a dit le 16 septembre 1986, dans le discours du Trône à l'ouverture de l'exercice parlementaire, qu'une réduction de la production agricole peut être favorable pour la nature et le paysage, et que la politique du gouvernement en matière de protection de la nature et du paysage sera poursuivie. 


\section{ADAPTATION DE L'ORGANISATION DE L'AFN}

L'Administration forestière néerlandaise (AFN) est donc un service public du ministère de l'Agriculture et de la Pêche, qui est responsable des tâches suivantes:

- application des lois concernant le domaine de la sylviculture et de la protection de la nature, y compris les "monuments verts" et sites naturels ;

- gestion des forêts domaniales, y compris les plantations domaniales hors forêts :

- gestion des réserves naturelles domaniales;

- vulgarisation des techniques auprès des propriétaires forestiers, de droit public ou privé, associations, fondations et sociètés :

- idem pour les réserves nationales;

- aménagement du paysage, y compris l'accompagnement des remembrements ruraux ;

- vulgarisation éducative du grand public.

L'organisation de l'AFN dans les provinces du pays donne la vue chronologique suivante:

- période 1945-1956: un seul ingénieur forestier dans chaque province, responsable et chargé de toutes les tâches de l'AFN;

- après 1956, la spécialisation des tâches étant accentuée, cela impliqua quatre ingénieurs par province pour la gestion des cantonnements, les tâches légales y compris la vulgarisation sylvicole, la protection de la nature et l'aménagement du paysage;

- en 1966, nomination d'un directeur provincial de l'AFN pour la coordination des quatre ingénieurs ;

- à partir de 1972, le directeur provincial fut placé sous la juridiction d'un directeur général; les tâches du directeur étaient augmentées des tâches suivantes: loi sur la chasse, la pêche et les politiques de génie rural, y compris le remembrement rural et l'acquisition des terres agricoles pour améliorer les structures agricoles. Tous les ingénieurs travaillent sous la juridiction de directeurs de directions différentes. Le directeur provincial est le coordinateur des ingénieurs de sa province;

- après 1984, nouvelle organisation: la fonction de génie rural et de remembrement fut supprimée, et le directeur provincial chargé d'une tâche nouvelle appelée "espaces verts et qualité du milieu à la campagne ". Chaque directeur restait sous la juridiction du directeur général.

Jusqu'en 1982, c'était le ministère des Affaires culturelles qui était responsable de la protection de la nature et des loisirs, mais les tâches de la protection de la nature étaient exécutées par l'AFN. En 1982, après les élections, le nouveau gouvernement a institué un ministère de l'Agriculture et de la Pêche incorporant dans ses responsabilités politiques la protection de la nature et les loisirs. Ces tâches ont été commises aux soins de deux nouvelles directions centrales au Ministère; par la suite, l'AFN a perdu la tâche de la conservation de la nature : cela implique un appauvrissement grave pour ce service.

En ce qui concerne la structure d'organisation (établie en 1972 et modifiée en 1984), les ingénieurs et autres hauts fonctionnaires du Ministère, qui sont décentralisés dans chaque province, ont été adjoints au directeur nommé pour la province. Ce directeur est responsable de véritables tâches de directeur général, il est le représentant du ministre dans sa province. Premièrement, le directeur possède la tâche de l'aménagement du territoire rural, inscrit aux plans des planifications urbaine et rurale. Ainsi les ingenieurs de l'AFN travaillent sous la juridiction du directeur général de l'AFN, mais ils sont aussi adjoints aux directeurs provinciaux. Ces directeurs sont chargés de la responsabilité de la coordination de toutes les tâches dans leur province au nom du ministre. Cette organisation a ses faiblesses: d'une part, les ingénieurs 
ont un directeur au plus haut niveau qui leur donne des directives "centrales"; d'autre part, le directeur provincial leur donne des instructions qui résultent de sa responsabilité de coordination.

En cas de situations conflictuelles, les deux directeurs (provincial et forestier) se concertent au niveau provincial et doivent résoudre les problèmes du conflit. Si une solution n'est pas possible, les directeurs doivent soumettre le problème au plus haut niveau qui prendra la décision. Cette organisation fait appel à une bonne entente entre le directeur provincial et son collègue le directeur de l'AFN (et essentiellement les autres directeurs). La même bonne entente est aussi nécessité urgente entre le directeur et les ingénieurs qui pratiquement lui sont adjoints. Formellement la relation d'autorité entre le directeur provincial, ses ingénieurs et les hauts fonctionnaires n'est pas fondèe sur l'organisation hiérarchique pure, mais elle dépend de l'attitude personnelle confraternelle de tous les fonctionnaires. Cette organisation demande beaucoup de consultations, de délibérations, et de compréhension mutuelle.

\section{CONCLUSIONS}

La progression générale des surfaces forestières, la progression des feuillus par rapport aux résineux, puis la prise en compte d'une foresterie à usages multiples (production de bois, loisirs, protection de la nature) ont donc, jusqu'en 1986, caractérise l'évolution positive de la forêt néerlandaise, et d'abord de ses forêts domaniales, données en exemple à toutes les autres.

Cette évolution traduit dans les faits l'intérêt de plus en plus marqué que, depuis 1960, le monde politique et les gouvernements ont portè à la forêt.

Depuis les années 80 , un pas nouveau a été franchi: il s'agit maintenant d'aménager de façon globale les espaces verts, forestiers ou non, au niveau provincial. Pari séduisant, mais parfois difficile. Sera-t-il gagné ? Nous le saurons dans un avenir proche.

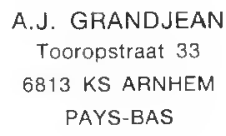

